\title{
Lonely SARTs: loneliness and sustained attention in the Irish longitudinal study of aging
}

Joanna E. McHugh Power, Caoimhe Hannigan, Sile Carney, Joanne Feeney, Rose Ann Kenny, Frank Kee \& Brian A. Lawlor

To cite this article: Joanna E. McHugh Power, Caoimhe Hannigan, Sile Carney, Joanne Feeney, Rose Ann Kenny, Frank Kee \& Brian A. Lawlor (2020) Lonely SARTs: loneliness and sustained attention in the Irish longitudinal study of aging, Aging, Neuropsychology, and Cognition, 27:2, 197-206, DOI: $10.1080 / 13825585.2019 .1602705$

To link to this article: https://doi.org/10.1080/13825585.2019.1602705

View supplementary material $\widetilde{ }$

Published online: 18 Apr 2019.

Submit your article to this journal

Џ Article views: 247

Q View related articles

View Crossmark data $\nearrow$ 


\title{
Lonely SARTs: Ioneliness and sustained attention in the Irish longitudinal study of aging
}

\author{
Joanna E. McHugh Power (D) ${ }^{a, b}$, Caoimhe Hannigan ${ }^{b, c}$, Sile Carneyc, Joanne Feeney ${ }^{c}$, \\ Rose Ann Kenny ${ }^{c}$, Frank Kee $\mathbb{D D}^{a}$ and Brian A. Lawlor
}

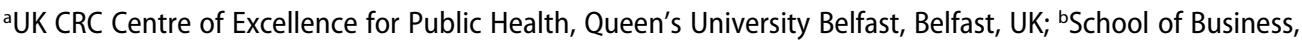
National College of Ireland, Dublin 1, Ireland; 'School of Nursing, School of Medicine, and School of Medical Gerontology, Trinity College, Dublin 2, Ireland

\begin{abstract}
Based on biologically plausible mechanisms and previous research, it is possible to hypothesize a reciprocal association between sustained attention and loneliness. We investigated this association using a cross-lagged modeling approach. Using data from 6,239 participants aged over 50 in TILDA, a nationally representative study of aging, we used structural equation models to investigate potential cross-lagged associations between sustained attention and loneliness, measured at baseline and again after four years. Sustained attention at baseline had a small association with loneliness four years later, but loneliness at baseline was not associated with sustained attention at follow-up. Auto-regressive associations were strong for both loneliness over time and sustained attention over time. Sustained attention may account for a small proportion of the variance in loneliness over time among older adults, and may constitute a risk factor in the development of loneliness. Implications for the identification of at-risk individuals and the prevention of loneliness are discussed.
\end{abstract}

\section{ARTICLE HISTORY}

Received 14 September 2018 Accepted 27 March 2019

\section{KEYWORDS}

Cognitive; cross-lagged; structural equation modelling; observational

\section{Introduction}

Loneliness is the felt inadequacy of social contact relative to that desired (L.A. Peplau \& Perlman, 1982), and has been shown to be associated with adverse health consequences for older adults (Holt-Lunstad, Smith, Baker, Harris, \& Stephenson, 2015; Valtorta, Kanaan, Gilbody, Ronzi, \& Hanratty, 2016). While most researchers investigate loneliness as being a cause of cognitive decline, it is also possible that cognitive declines play a role in the etiology of loneliness (de Jong Gierveld, 1987; Fees, Martin, \& Poon, 1999). For instance, superior cognitive functioning may in some way protect against loneliness in later life (Martin, Hagberg, \& Poon, 1997). More recently, poor baseline cognitive functioning has been shown to predict higher levels of loneliness over time in older American adults (Donovan et al., 2017), potentially because cognitive decline might cause social withdrawal (Charles \& Carstensen, 2010; Crooks, Lubben, Petitti, Little, \& Chiu, 2008), potentially precipitating loneliness.

It is worth considering whether specific cognitive domains predict loneliness. Because of the multidimensional natures of both social and cognitive function, there is a need for 
more domain-specific research that can explore connections between the two in a more granular manner (House, Umberson, \& Landis, 1988; Krueger et al., 2009). One domainspecific relationship worth investigating is that between attention and loneliness. Having disorders of attention such as Attentional Deficit and Hyperactivity Disorder (ADHD) was associated with increased loneliness among older adults (Michielsen et al., 2015) since, as the authors comment, inattention may make social interaction difficult. A cross-sectional association between attention and loneliness exists (Tzang, Yang, Yeh, Liu, \& Tsai, 2015), while lonely individuals have been shown to have poor attentional performance (Letitia A Peplau, Perlman, Peplau, \& Perlman, 1982).

The directionality of the causal pathway between loneliness and attention is not yet well established. Social attention difficulties in particular might drive loneliness (Conroy, Golden, Jeffares, O'Neill, \& McGee, 2010), as may hyper-vigilance to social threat (Bangee, Harris, Bridges, Rotenberg, \& Qualter, 2014; Cacioppo, Norris, Decety, Monteleone, \& Nusbaum, 2009; Gardner, Pickett, Jefferis, \& Knowles, 2005).

More specifically, we recognize the need to focus more on the potential relationship between sustained attention and loneliness. Sustained attention refers to the ability to mindfully sustain the processing of stimuli that do not inherently capture attention (Robertson, Manly, Andrade, Baddeley, \& Yiend, 1997). While it is debatable whether the aging process itself impacts on the ability to sustain attention (Staub, Doignon-Camus, Despres, \& Bonneford, 2013), sustained attention underlies other cognitive functions that are vulnerable to age-related decline (Sarter, Givens, \& Bruno, 2001).

Preliminary neuropsychological evidence suggests that neural mechanisms of sustained attention may underlie loneliness. Activation in nodes of the ventral attention network, which reorients attention to exogenous cues, and is suppressed during goaldirected, top-down tasks which require the maintenance of attention (Corbetta, Patel, \& Shulman, 2008; Tian, Liang, \& Yao, 2014), is associated with higher levels of loneliness (Tian et al., 2014). Tian and colleagues interpret their findings to mean that loneliness may, at a neural level, reflect excessive reorientation to potential threats in the environment, at the cost of sustaining top-down attentional processes. Thus it is possible, based on Tian's findings, to hypothesize that loneliness is associated with a failure to suppress the ventral attention network, manifesting in poor performance at sustained attention tasks. This hypothesis would also account for Cacioppo's finding that loneliness involves hyper-vigilance for social threats (Cacioppo et al., 2009).

It is equally possible to hypothesize that loneliness drives poor sustained attention, since loneliness is most typically found to be a predictor of cognitive decline more generally. High levels of loneliness are also associated with cognitive decline (Boss, Kang, \& Branson, 2015; Cacioppo \& Cacioppo, 2013; Ellwardt, Aartsen, Deeg, \& Steverink, 2013), as well as increased risk of dementia (Holwerda et al., 2014; Wilson et al., 2007), and may even constitute an early marker of Alzheimer's disease (Donovan et al., 2016). Across a sample of breast cancer survivors and healthy controls, Jaremka et al. (2014) found that lonely participants had worse scores on a measure of sustained concentration (Homack \& Riccio, 2006), and attributed this performance to pre-existing loneliness as a risk factor. However, their design precluded comment on the directionality of the relationship between loneliness and sustained concentration.

We investigated whether sustained attention is associated with loneliness after controlling for covariates, in a sample of healthy older adults participating in the Irish Longitudinal Study 
of Ageing (TILDA). We used a cross-lagged panel modeling approach, to additionally model the possibility that the hypothesized association between loneliness and sustained attention is the other way around, such that loneliness predicts sustained attention, in the same way that it predicts global cognitive function (Boss et al., 2015). We hypothesized a bi-directional relationship: that sustained attention would predict loneliness, and the converse, that loneliness would predict sustained attention.

\section{Methods}

\section{Design}

The current analysis utilized data from waves 1 and 3 of the Irish Longitudinal Study of Ageing (TILDA), which were spaced four years apart. TILDA is a nationally representative, prospective, longitudinal cohort study in the Republic of Ireland. TILDA assesses social, economic, and health circumstances among 8175 community-dwelling adults aged over 50 at wave 1 (more information can be found on the study design of TILDA at (Kenny et al., 2010)). The study was approved by the faculty of health sciences research ethics committee at Trinity College Dublin. Participants were recruited to TILDA using a random sampling procedure based on geographic location, with additional inclusion criteria of being 50 years of age or over, and resident in Ireland. The sample size required to represent the national aging population was 8,000 (Kenny et al., 2010). If eligible participants were married or partnered, their spouses and partners (of any age) were also invited to take part.

\section{Participants}

The current analyses relate to 6,239 participants of the TILDA study who were aged over 50 (thus excluding some partners of participants who were under 50 ). This sample was 45.4\% male and $54.6 \%$ female (see Table 1 ).

\section{Measures - covariates}

Within the cross-lagged models, analyses controlled for the following covariates: age, sex (dummy coded such that $0=$ male and $1=$ female), education (assessed as a three-level

Table 1. Baseline sample characteristics for the 6,239 participants from the TILDA study whose data are analyzed in the current investigation (CESD20: the 20-item Centre for Epidemiological Studies depression scale; CESDNoLon: CESD20 with item enquiring about loneliness removed; SART RT: Sustained Attention to Response Task Reaction Times).

\begin{tabular}{lc}
\hline & Mean, SD (or percentages where appropriate) \\
\hline Age & $63.05(9.22)$ \\
Sex & $54.6 \%$ females; $45.4 \%$ males \\
Education & $26.96 \%$ no qualification \\
& $41.07 \%$ intermediate qualification \\
CESD20 & $31.96 \%$ degree qualification or higher \\
CESDNoLon & $5.68(7.07)$ \\
Loneliness & $5.41(6.69)$ \\
Coefficient of variation SART & $1.07(1.45)$ \\
Commission errors SART & $0.31(0.16) ;$ median $=0.28$ \\
\hline
\end{tabular}


variable where levels were "no qualification", "intermediate qualification", and "degree qualification or higher"), and depressive symptomatology (scores on the Centre for Epidemiological Studies Depression scale (Radloff, 1977) with the item inquiring about loneliness removed).

\section{Sustained attention}

Sustained attention was assessed using the Sustained Attention to Response Task (SART; Robertson et al., 1997). In this task, a series of single digits between 1 and 9 are sequentially presented to the participant on a computer screen. Each digit is presented for 300 ms, with 800 ms intervals between digits. Participants are instructed to press a keyboard key as soon as possible (with response time noted) for each digit presented, except if the digit is 3 . For the purposes of the current analysis, a latent "sustained attention" variable was created with two indicators: (a) the coefficient of variation of the reaction times to the SART, a measure of performance variability and (b) the number of commission errors made by each participant, or the number of trials in which the participant pressed the response key following the presentation of the digit " 3 '. These two indicators were chosen following consultation with the lead author of the original task (Robertson et al., 1997). Trials in which response times were outside of three standard deviations of the participants" mean response time were defined as outliers and removed since they likely reflect fatigue, loss of interest or test interruption.

\section{Loneliness}

Loneliness was specified as a latent factor with three indicators: the items constituting the 3-item version of the University of California, Los Angeles (UCLA) Loneliness scale (Russell, 1996). This scale has previously been shown to have acceptable psychometric characteristics (Hughes, Waite, Hawkley, \& Cacioppo, 2004; Russell, 1996). Its three items are "I feel left out", "I feel isolated", and "I lack companionship".

\section{Data analysis}

Data from participants aged over $50(n=6,239)$ only were included for the purposes of this analysis. Data were all tested for multivariate normality and assumptions for regression were tested and satisfied. We initially specified a model within an SEM framework and using a maximum likelihood estimator (using the lavaan package; Rosseel, 2012) with auto-regressive pathways and covariates only, and in a second model, included cross-lagged paths such that loneliness at wave 3 was regressed on sustained attention at wave 1, and sustained attention at wave 3 was regressed on loneliness at wave 1 . In accordance with the approach previously laid out (Hyland et al., 2015) we conducted these two models and compared them using Akaike and Bayesian Information Criteria (whereby smaller values indicate better model fit). If in model 2, then, there are crosslagged paths between sustained attention and loneliness, a third optional step is to constrain the two cross-lagged paths as equal; if this third model is a better fit to the data than model 2 (in which both paths are freely estimated), one can conclude that there is no single dominant pathway in the cross-lagged association. Step 3 is not deemed to be necessary if, at step 2, there is an absence of cross-lagged paths. 
While AIC and BIC can provide relative evaluation of models, absolute model fit was evaluated using standard SEM indices - the confirmatory fit index (CFI), Tucker Lewis index (TLI), root mean square error of approximation (RMSEA) and standardized root mean square residuals (SRMR). Both the CFI and TLI should be above 0.9, while the RMSEA should be lower than 0.05, and the SRMR lower than 0.08 (Hu \& Bentler, 1999).

\section{Results}

Average age was 63.05 (50-105 age range, SD =9.22). For the most part, participants had low levels of depressive, anxiety, and loneliness symptoms (see Table 1). Analyses were conducted on data pertaining to those individuals who took part in the comprehensive health assessment only.

\section{Model 1: autoregressive paths}

The model converged normally after 182 iterations, and fit was marginally acceptable, $\mathrm{X}_{417}^{2}=5683.68, \mathrm{p}<0.001, \mathrm{CFI}=0.89, \mathrm{TLI}=0.89, \mathrm{RMSEA}=0.045\left(\mathrm{Cl}_{90}=0.044,0.046\right)$, $\mathrm{SRMR}=0.046$. The measurement model component indicated that items loaded on satisfactorily to the three latent variables of depression, loneliness, and sustained attention (see Supplementary Materials, Table 1).

Looking to the structural component of the model, then, clear auto-regressive paths were evident between waves 1 and 3 of data collection for both sustained attention, $\beta=0.8$, $p<0.001$, and loneliness, $\beta=0.62, p<0.001$. Higher age, female sex, lower education, and higher levels of depressive symptomatology all predicted worse performance on the SART, while male sex, higher education, and higher depression levels all predicted higher levels of loneliness (see Table 2). Notably, age at wave 1 did not predict loneliness at wave 3.

\section{Model 2: cross-lagged paths}

Next, a second model with cross-lagged paths (from SART at wave 1 to Loneliness at wave 3, and from Loneliness at wave 1 to SART at wave 3) was conducted. Again the model converged normally, after 230 iterations, and fit was marginally acceptable, $x^{2}{ }_{415}=5676.97, p<0.001$,

Table 2. Structural model component of model 1: autoregressive paths only.

\begin{tabular}{lrrrr}
\hline & Beta & SE & $Z$ & $p$ \\
\hline SART Wave 3 as outcome & & & & \\
SART Wave 1 & 0.80 & 0.15 & 9.89 & $<0.001$ \\
Age & 0.22 & 0.01 & 7.43 & $<0.001$ \\
Sex & 0.05 & 0.07 & 2.89 & 0.004 \\
Education & -0.11 & 0.05 & -5.25 & $<0.001$ \\
Depression & 0.05 & 0.04 & 2.22 & 0.03 \\
Loneliness Wave 3 as outcome & & & & \\
Loneliness Wave 1 & 0.84 & 0.03 & 28.21 & $<0.001$ \\
Age & -0.00 & 0.00 & -0.23 & 0.818 \\
Sex & -0.07 & 0.03 & -2.09 & 0.04 \\
Education & 0.05 & 0.02 & 2.32 & 0.02 \\
Depression & -0.15 & 0.02 & -6.87 & $<0.001$ \\
\hline
\end{tabular}


Table 3. Structural model component of model 1: cross-lagged paths included.

\begin{tabular}{lccrr}
\hline & Beta & SE & $Z$ & $p$ \\
\hline SART Wave 3 as outcome & & & & \\
Loneliness Wave 1 & 0.02 & 0.05 & -0.64 & 0.520 \\
SART Wave 1 & 0.79 & 0.15 & 9.90 & $<0.001$ \\
Age & 0.22 & 0.01 & 7.43 & $<0.001$ \\
Sex & 0.05 & 0.07 & 2.91 & 0.004 \\
Education & -0.11 & 0.05 & -5.26 & $<0.001$ \\
Depression & 0.04 & 0.05 & 1.55 & 0.121 \\
Loneliness Wave 3 as outcome & & & \\
SART Wave 1 & 0.05 & 0.03 & -2.53 & 0.01 \\
Loneliness Wave 1 & 0.61 & 0.03 & 28.05 & $<0.001$ \\
Age & 0.01 & 0.00 & 0.43 & 0.67 \\
Sex & -0.02 & 0.03 & -1.91 & 0.06 \\
Education & 0.03 & 0.02 & 1.89 & 0.06 \\
Depression & -0.11 & 0.02 & -6.82 & $<0.001$ \\
\hline
\end{tabular}

$\mathrm{CFI}=0.89, \mathrm{TLI}=0.89, \mathrm{RMSEA}=0.045\left(\mathrm{Cl}_{90}=0.044,0.046\right), \mathrm{SRMR}=0.045$. The association between Loneliness at wave 1 and SART at wave 3 was not significant, $\beta=-0.02, p=0.52$, but the association between SART at wave 1 and Loneliness at wave 3 was significant and larger, $\beta=-0.05, p=.01$, indicating that as performance in SART deteriorates, loneliness increases (see Table 3). Since only one path of the two potential cross-lagged paths demonstrated significance, there was no need to conduct step 3 (to explore whether one path was dominant over the other).

\section{Discussion}

The study aimed to evaluate cross-lagged associations between loneliness and sustained attention in a large sample of adults over 50. We hypothesised that there would be a reciprocal relationship between sustained attention and loneliness. While sustained attention at baseline predicted loneliness four years later, the converse, that loneliness would predict sustained attention, was not supported. Importantly, auto-regressive paths were strong such that the best predictor of sustained attention at follow-up was sustained attention at baseline, and the same pattern was observed for loneliness. The effect sizes of the cross-lagged paths were both small.

Results in relation to the impact of loneliness on sustained attention are difficult to integrate with previous literature. Since prior findings demonstrate consistently that loneliness precedes cognitive decline (Boss et al., 2015; Cacioppo \& Cacioppo, 2013; Ellwardt et al., 2013), one might expect that it would also precede declines in cognitive domains.

In fact, our pattern of results closely mimics those previously reported by Ayalon and colleagues in relation to the reciprocal relationships between loneliness and memory (Ayalon, Shiovitz-Ezra, \& Roziner, 2016). In their study, Ayalon reported that while memory predicted loneliness over time, loneliness did not predict memory. Ayalon's analysis utilized data collected from older participants in the Health and Retirement Study, with which the TILDA study is harmonized, and the follow-up timeframe involved was the same as that in the current study (i.e., four years). Since the studies are not 
sufficiently similar to allow a direct comparison, it would be of interest for future studies to evaluate comparative effects of different cognitive domains in predicting loneliness. Boss and colleagues conducted a systematic review of the literature and concluded that loneliness was predictive of cognitive domains such as intellectual functioning, processing speed, and memory, but attention was not a domain considered specifically in any of the studies they reviewed (Boss et al., 2015).

Our findings indicate that loneliness is in small part predicted by sustained attention. As performance in sustained attention increases, loneliness at a four-year follow-up decreases among our sample. This finding corroborates our hypothesis in part and is in accordance with previous literature suggesting a role for better cognitive functioning in predicting attenuated feelings of loneliness (de Jong Gierveld, 1987; Donovan et al., 2017; Fees et al., 1999; Martin et al., 1997). Findings also accord with a previous finding of a cross-sectional association between attention and loneliness (Tzang et al., 2015). Results also make sense in the context of previous research which has suggested that individuals who are lonely have tendencies to divert attention to social cues with excessive vigilance (Bangee et al., 2014; Cacioppo et al., 2009; Gardner et al., 2005), a feature or behaviour that can be differentiated from a more sustained form of attention (Corbetta et al., 2008; Tian et al., 2014, 2014). Increased vigilance and distractibility in the presence of social cues would necessarily lead to a decrease in performance in tasks of sustained attention to non-social stimuli, in keeping with these previous suggestions by Corbetta, Tian, and colleagues (ibid.)

The current study is not without limitations. We frame our analysis as an explanatory rather than a predictive model and as such it was our aim to test a causal hypothesis rather than create the most accurate prediction of either loneliness or sustained attention (Shmueli, 2010). We used the standard SEM indices to evaluate absolute model fit, but these indices are not without issue and must be considered as a supplement to the magnitude and statistical significance of the model coefficients (Newsom, 2016).

It is possible that the model failed to account for an important confounders in the association between sustained attention and loneliness. We controlled for age, gender, education level, and depressive symptomatology in the current analysis, which represent, to our understanding, the principle areas of interest when evaluating the relationship between sustained attention and loneliness. However, it is possible that our covariate coverage and analysis strategy was too narrow or misplaced. For instance, since it has been suggested that sustained attention underlies cognitive processes (Staub et al., 2013) perhaps cognitive decline in fact mediates some of the association between sustained attention and loneliness. Future research employing mediation models would be able to evaluate this possibility. It is equally possible that we have not adequately explored other potential mediators in the relationship between sustained attention and loneliness. Some previous research indicates that individuals with attention disorders can report higher than average levels of loneliness (Michielsen et al., 2015) which may be due to difficulties in maintaining social interactions. Our current findings accord with this purported mechanism since we note that sustained attention is associated with loneliness. Additional analyses are required to further explore the possibility that social interaction may mediate the association between sustained attention at baseline and loneliness at follow-up. 
The value of the current study lies in its attempt to tackle a relatively neglected research question - the association between domains of cognitive function and loneliness (House et al., 1988; Krueger et al., 2009). We report that sustained attention difficulties may predict loneliness among older adults. Results have clinical implications for those looking to prevent loneliness in later life, which has of late become a serious public health priority (Hunter, 2012; Klinenberg, 2016), notably within the UK in the Campaign to End Loneliness, and the more recent Loneliness Taskforce established in the Republic of Ireland. While effect sizes were small, it should be noted that sustained attention represents one risk factor for the development of loneliness over time. One way in which loneliness might be avoided is by supporting the maintenance of functioning in specific cognitive domains such as sustained attention. Taken together with Ayalon's finding of an effect of memory on loneliness, it could be suggested that domain-specific focus in cognitive maintenance in later life might represent a useful strategy to mitigate or avoid loneliness in this population.

In summary, we report that while sustained attention has a small association with loneliness at follow-up, loneliness did not predict sustained attention at follow-up.

\section{Acknowledgments}

The authors wish to thank members of the TILDA team who gave critical review to the manuscript during its preparation, and particularly to Professor lan Robertson, School of Psychology, Trinity College Dublin.

\section{Disclosure statement}

No potential conflict of interest was reported by the authors.

\section{Funding}

The study was supported by a grant received by the corresponding author from the Centre for Ageing Research and Development in Ireland, in the form of a Leadership in Ageing Research Fellowship. TILDA is funded by Atlantic Philanthropies, Irish Life, and the Department of Health in Ireland.

\section{ORCID}

Joanna E. McHugh Power (D) http://orcid.org/0000-0002-7387-3107

Frank Kee (D) http://orcid.org/0000-0002-0606-8167

\section{References}

Ayalon, L., Shiovitz-Ezra, S., \& Roziner, I. (2016). A cross-lagged model of the reciprocal associations of loneliness and memory functioning. Psychology and Aging, 31(3), 255-261.

Bangee, M., Harris, R., Bridges, N., Rotenberg, K. J., \& Qualter, P. (2014). Loneliness and attention to social threat in young adults: Findings from an eye tracker study. Personality and Individual Differences, 63, 16-23.

Boss, L., Kang, D. H., \& Branson, S. (2015). Loneliness and cognitive function in the older adult: A systematic review. International Psychogeriatrics, 27(4), 541-553. 
Cacioppo, J. T., \& Cacioppo, S. (2013). Older adults reporting social isolation or loneliness show poorer cognitive function 4 years later. Evidence Based Nursing. doi:10.1036/eb-2013101379

Cacioppo, J. T., Norris, C. J., Decety, J., Monteleone, G., \& Nusbaum, G. (2009). In the eye of the beholder: Individual differences in perceived social isolation predict regional brain activation to social stimuli. Journal of Cognitive Neuroscience, 21, 83-92.

Charles, S. T., \& Carstensen, L. L. (2010). Social and emotional aging. Annual Review of Psychology, 61, 383-409.

Conroy, R. M., Golden, J., Jeffares, I., O'Neill, D., \& McGee, H. (2010). Boredom-proneness, loneliness, social engagement and depression and their association with cognitive function in older people: A population study. Psychology, Health and Medicine, 15(4), 463-473.

Corbetta, M., Patel, G., \& Shulman, G. L. (2008). The reorienting system of the human brain: From environment to theory of mind. Neuron, 58, 306-324.

Crooks, V. C., Lubben, J., Petitti, D. B., Little, D., \& Chiu, V. (2008). Social network, cognitive function, and dementia incidence among elderly women. American Journal of Public Health, 98(7), $1221-1227$.

de Jong Gierveld, J. (1987). Developing and testing a model of loneliness. Journal of Personality and Social Psychology, 53(1), 119-128.

Donovan, N. J., Okereke, O. I., Vannini, P., Amariglio, R. E., Rentz, D. M., Marshall, G. A., ... Sperling, R. A. (2016). Association of higher cortical amyloid burden with loneliness in cognitively normal older adults. JAMA psychiatry, 73(12), 1230-1237.

Donovan, N. J., Wu, Q., Rentz, D. M., Sperling, R. A., Marshall, G. A., \& Glymour, M. M. (2017). Loneliness, depression and cognitive function in older US adults. International Journal of Geriatric Psychiatry, 32(5), 564-573.

Ellwardt, L., Aartsen, M., Deeg, D., \& Steverink, N. (2013). Does loneliness mediate the relation between social support and cognitive functioning in later life? Social Science \& Medicine, 98, $116-124$.

Fees, B., Martin, P., \& Poon, L. W. (1999). A model of loneliness in older adults. Journal of Gerontology B: Psychological Sciences \& Social Sciences, 54(4), 231-239.

Gardner, W. L., Pickett, C. L., Jefferis, V., \& Knowles, M. (2005). On the outside looking in: Loneliness and social monitoring. Personality and Social Psychology Bulletin, 31(11), 1549-1560.

Holt-Lunstad, J., Smith, T. B., Baker, M., Harris, T. B., \& Stephenson, D. (2015). Loneliness and social isolation as risk factors for mortality: A meta-analytic review. Perspectives on Psychological Science, 10, 227-237.

Holwerda, T. J., Deeg, D., Beekman, A. T. F., van Tilburg, T. G., Stek, M. L., Jonker, C., \& Schoevers, R. A. (2014). Feelings of loneliness, but not social isolation, predict dementia onset: Results from the Amsterdam Study of the Elderly (AMSTEL). Journal of Neurology, Neurosurgery \& Psychiatry, 85, 135-142.

Homack, S., \& Riccio, C. A. (2006). Conners' Continuous Performance test (2nd Ed: CCPT-II). Journal of Attention Disorders, 9, 556-558.

House, J. S., Umberson, D., \& Landis, K. R. (1988). Structures and processes of social support. Annual Review of Sociology, 14, 293-318.

Hu, L., \& Bentler, P. M. (1999). Cutoff criteria for fit indexes in covariance structure analysis: Conventional criteria versus new alternatives. Structural Equation Modeling: A Multidisciplinary Journal, 6(1), 1-55.

Hughes, M. E., Waite, L. J., Hawkley, L. C., \& Cacioppo, J. T. (2004). A short scale for measuring loneliness in large surveys results from two population-based studies. Research on Aging, 26(6), 655-672.

Hunter, D. (2012). Loneliness: A public health issue. Perspectives in Public Health, 132(4), 153.

Hyland, P., Shevlin, M., Hansen, M., Vallieres, F., Murphy, J., \& Elklit, A. (2015). The temporal relations of PTSD symptoms among treatment-seeking victims of sexual assault: A longitudinal study. Journal of Loss and Trauma, 21(6), 492-506. 
Jaremka, L. M., Peng, J., Bornstein, R., Alfano, C., Andridge, R. R., Povoski, S. P., ... Kiecolt-Glaser, J. K. (2014). Cognitive Problems among breast cancer survivors: Loneliness enhances risk. Psychooncology, 23(12), 1356-1364.

Kenny, R. A., Whelan, B. J., Cronin, H., Kamiya, Y., Kearney, P., O'Regan, C., \& Ziegel, M. (2010). The design of the Irish longitudinal study on ageing. Dublin: Trinity College Dublin.

Klinenberg, E. (2016). Social isolation, loneliness, and living alone: Identifying the risks for public health. American Journal of Public Health, 106(5), 786-787.

Krueger, K. R., Wilson, R. S., Kamenetsky, J. M., Barnes, L. L., Bienias, J. L., \& Bennett, D. A. (2009). Social engagement and cognitive function in old age. Experimental Aging Research, 35(1), 45-60.

Martin, P., Hagberg, B., \& Poon, L. W. (1997). Predictors of loneliness in centenarians: A parallel study. Journal of Cross-Cultural Gerontology, 12, 203-224.

Michielsen, M., Comijs, H. C., Aartsen, M. J., Semeijn, E. J., Beekman, A. T. F., Deeg, D., \& Kooij, J. J. (2015). The relationships between ADHD and social functioning and participation in older adults in a population based study. Journal of Attention Disorders, 19(5), 368-379.

Newsom, J. T. (2016). Cross-lagged panel analysis. In S. Krauss Whitbourne (Ed.), The encyclopedia of adulthood and aging. Wiley Online. http://onlinelibrary.wiley.com/doi/10.1002/ 9781118521373.wbeaa250/full

Peplau, L. A., \& Perlman, D. (1982). Loneliness: A sourcebook of curent theory, research, and therapy. New York: Wiley.

Peplau, L. A., Perlman, D., Peplau, L. A., \& Perlman, D. (1982). Perspectives on loneliness. In A. Peplau and D. Perlman (Eds.), Loneliness: A sourcebook of current theory, research and therapy (pp. 1-20). Michigan, USA: Wiley.

Radloff, L. (1977). The CES-D Scale: A self report depression scale for research in the general population. Applied Psychological Measurement, 1, 385-401.

Robertson, I. H., Manly, T., Andrade, J., Baddeley, B. T., \& Yiend, J. (1997). 'Oops!' performance correlates of everyday attentional failures in traumatic brain injured and normal subjects. Neuropsychologia, 35(6), 747-758.

Rosseel, Y. (2012). lavaan: An R package for structural equation modeling. Journal of Statistical Software, 48(2), 1-36.

Russell, D. W. (1996). UCLA Loneliness Scale (Version 3): Reliability, validity, and factor structure. Journal of Personality Assessment, 66(1), 20-40.

Sarter, M., Givens, B., \& Bruno, J. P. (2001). The cognitive neuroscience of sustained attention: Where top-down meets bottom up. Brain Research Reviews, 35, 146-160.

Shmueli, G. (2010). To explain or to predict? Statistical Science, 25(3), 289-310.

Staub, B., Doignon-Camus, N., Despres, O., \& Bonneford, A. (2013). Sustained attention in the elderly: What do we know and what does it tell us about cognitive aging? Ageing Research Reviews, 12(2), 459-468.

Tian, Y., Liang, S., \& Yao, D. (2014). Attentional orienting and response inhibition: Insights from spatial-temporal neuroimaging. Neuroscience Bulletin, 30, 141-152.

Tian, Y., Liang, S., Yuan, Z., Chen, S., Xu, P., \& Yao, D. (2014). White matter structure in loneliness: Preliminary findings from diffusion tensor imaging. NeuroReport, 25, 843-847.

Tzang, R., Yang, A., Yeh, H., Liu, M., \& Tsai, S. (2015). Association of loneliness and depression with specific cognitive performance in non-demented elderly males. Medical Science Monitor, 21, 100-104.

Valtorta, N. K., Kanaan, M., Gilbody, S., Ronzi, S., \& Hanratty, B. (2016). Loneliness and social isolation as risk factors for coronary heart disease and stroke: Systematic review and meta-analysis of longitudinal observational studies. Heart. doi:10.1136/heartjnl-2015-308790

Wilson, R. S., Krueger, K. R., Arnold, S. E., Schneider, J. A., Kelly, J. F., Barnes, L. L., ... Bennett, D. A. (2007). Loneliness and risk of Alzheimer disease. Archives of General Psychiatry, 64, 234-240. 